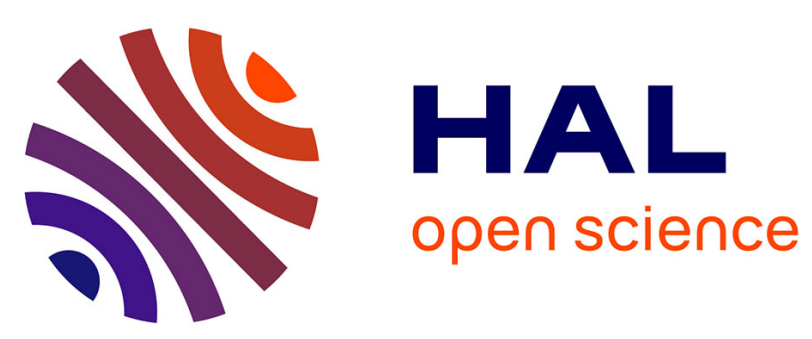

\title{
EXFOLIATION DU GRAPHITE PAR TRAITEMENT LASER D'UN COMPOSÉ INTERCALAIRE
}

\author{
A. Thomy, J. Ousset, G. Furdin, Jean Pelletier, A. Vannes
}

\section{To cite this version:}

A. Thomy, J. Ousset, G. Furdin, Jean Pelletier, A. Vannes. EXFOLIATION DU GRAPHITE PAR TRAITEMENT LASER D'UN COMPOSÉ INTERCALAIRE. Journal de Physique Colloques, 1987, 48 (C7), pp.C7-115-C7-118. 10.1051/jphyscol:1987719 . jpa-00227015

\section{HAL Id: jpa-00227015 https://hal.science/jpa-00227015}

Submitted on 1 Jan 1987

HAL is a multi-disciplinary open access archive for the deposit and dissemination of scientific research documents, whether they are published or not. The documents may come from teaching and research institutions in France or abroad, or from public or private research centers.
L'archive ouverte pluridisciplinaire HAL, est destinée au dépôt et à la diffusion de documents scientifiques de niveau recherche, publiés ou non, émanant des établissements d'enseignement et de recherche français ou étrangers, des laboratoires publics ou privés. 


\section{EXFOLIATION DU GRAPHITE PAR TRAITEMENT LASER D'UN COMPOSÉ INTERCALAIRE}

A. THOMY, J.C. OUSSET, G. FURDIN* , J.M. PELLETIER** et A.B. VANNES $* *$

Laboratoire Mixte, CNRS Saint-Gobain, UM 37, CRPAM, BP 28 , F-54703 Pont-a-Mousson Cedex, France "Laboratoire de Chimie du Solide Minéral, Université Nancy $I$, CNRS UA-158, BP 239, F-54506 Vandoeuvre-1es-Nancy Cedex, France * GEMPPM, Bâtiment 502, ENSA Lyon, F-69621 Villeurbanne Ceđex, France *** Centre d'Application des Lasers de Forte Energie à la Transformation des Matériaux, INSA, Bâtiment 403. F-69621 Villeurbanne Cedex, France

Résume - L'exfoliation de graphite a êté réalisée par chauffage d'un composé intercalaire (graphite naturel-acide nitrique) au moyen d'un laser continu à $\mathrm{CO}_{2}$ et d'un laser YAG à impulsions. Les surfaces résultantes sont homogènes et nettement plus étendues que celles obtenues avec le même composé intercalaire par les procédés habituels de chauffage. Les expériences font ressortir l'intérêt du laser dans la recherche des conditions optimales d'exfoliation du graphite et dans les études visant à une meilleure compréhension du phénomène lui-même ; elles montrent clairément l'importance du choc thermique.

Abstract - For the first time graphite has been exfoliated by laser heating of an intercalated compound (natural graphite-nitric acid). A continuous $\mathrm{CO}_{2}$ laser and a pulsed YAG laser were used. Homogeneous surfaces were prepared which are larger than those obtained by a conventional heating process with the intercalated compound considered. Laser heating appears quite suitable for a better understanding of the exfoliation mechanism itself and for the finding of the best exfoliation conditions. The importance of the thermal shock is clearly evidenced.

\section{INTRODUCTION}

On entend par exfoliation du graphite la séparation plus ou moins complète de ses feuillets. La méthode la plus utilisée aujourd'hui consiste à décomposer thermiquement des composés intercalaires obtenus avec du graphite naturel. C'est ainsi que l'on procède en laboratoire pour obtenir des poudres de surface homogène particulièrement appropriées à l'étude des films bidimensionnels (cf.(1)). C'est également la méthode utilisée par l'industrie pour fabriquer le graphite exfolié qui est ensuite transformé par compression et laminage en feuilles qui sont commercialisées sous .1'appellation de Grafoil ou Papyex par les Sociétés "Union Carbide" et "Le Carbone Lorraine" respectivement (cf. brevets déposés à partir de 1968).

Jusquici, l'exfoliation de composés intercalaires du graphite a toujours été réalisée dans des fours classiques ou à induction et dans des flammes (cf.articles (2) - (13) qui traitent également des modifications des propriêtés superficielles du graphite dues à son exfoliation). 
L'étude exposée dans le présent article a été effectuée au moyen de lasers. Elle a pour but de montrer l'intérêt du chauffage laser pour la recherche des conditions optimales d'exfoliation d'un composé intercalaire donné en vue d'obtenir de grandes surfaces spécifiques.

\section{LE GRAPHITE ET LE COMPOSE INTERCALAIRE}

1) Le graphite de départ est une poudre de graphite naturel de Madagascar purifié (taux de cendres : $1 \%$ ) de $0,4 \mathrm{~m} 2 / \mathrm{g}$ d'aire spécifique et dont $80 \%$ des grains ont un diamètre équivalent compris entre 0,2 et $1 \mathrm{~mm}$.

2) Le composé intercalaire est un composé de stade 2 obtenu avec l'acide nitrique. Sa formule est $\mathrm{C}_{10}$, $\mathrm{HNO}_{3}$ (voir $(14,15$ ) pour une revue générale sur les composés intercalaires).

Ce composé a été choisi pour les raisons suivantes :

- sa synthèse est facile à réaliser et il a été bien caractérisé par des analyses radiocristallographiques $(16,17)$;

- son exfoliation par des procédés de chauffage conventionnels a déjà fait l'objet d'études $(18-20)$;

- enfin, ce composé est suffisamment stable pour pouvoir être manipulé à l'air à température ambiante.

Les différents échantillons avaient été conditionnés dans des ampoules scellées qui ne furent ouvertes qu'au moment des expériences.

\section{TRAITEMENTS THERMIQUES PAR LASERS}

\section{1) Lasers utilisés}

Les traitements ont été effectués au moyen de deux lasers :

- un laser à gaz $\left(\mathrm{CO}_{2}\right)$ utilisé en continu de type CI 4000 (Société ClLAS) ayant les caractéristiques suivantes : longueur d'onde $=10,6 \mu \mathrm{m}$; puissance réglable entre environ $120 \mathrm{~W}$ et $3600 \mathrm{~W}$ (en sortie de cavité).

- un laser à solide (YAG) à impulsions de type LAP 300 (Société Laser Applications) avec pour caractéristiques : longueur d'onde $=1,06 \mu \mathrm{m}$; impulsions. réglables entre 0,2 et $20 \mathrm{~ms}$; fréquence des tirs : de 1 à $300 \mathrm{~Hz}$; puissance moyenne : jusqu'à $300 \mathrm{~W}$, énergie par pulse (en sortie de cavité) $=40 \mathrm{~J}$ maximum.

(Pour plus ample information sur l'utilisation des lasers, voir (21, 22$)$ ).

\section{2) Expériences réalisées}

Huit échantillons du composé intercalaire considéré ont été traités :

- soit en les faisant défiler sous le faisceau du laser $\mathrm{CO}_{2}$ (échantillons 1-6) ou du laser YAG (échantillon 7),

- soit en les exposant à des pulses du laser YAG dans un tube à essai (éch.8 et 9)

Quand l'échantillon défilait sous le faisceau, il était disposé sur un support de graphite pour électrodes sur une largeur de $5 \mathrm{~mm}$, soit, dans tous les cas, la moitié du diamètre du faisceau au niveau de l'échantillon (10 mm). Ceci garantissait une bonne uniformité énergétique du rayonnement interagissant avec l'échantilion. Dans les traitements effectués dans un tube à essai quelques particules seulement du composé intercalaire etaient disposées au fond du tube. 
Les conditions dans lesquelles les expériences d'exfoliation ont été réalisées sont fournies tableau 1.

TABLEAU 1 - Conditions d'exfoliation et aires spécifiques obtenues (exfoliation conditions and resulting specific surface area)

\begin{tabular}{|c|c|c|c|c|c|}
\hline $\operatorname{Ech} \quad\left(\mathrm{CO}_{2}\right)$ & $P\left(W / \mathrm{cm}^{2}\right)$ & $V(\mathrm{~cm} / \mathrm{s})$ & \multicolumn{2}{|c|}{$t(s)$} & A $\left(\mathrm{m}^{2} / \mathrm{g}\right)$ \\
\hline 1 & 140 & 10 & \multicolumn{2}{|c|}{0,1} & 52,5 \\
\hline 2 & 140 & 0,5 & \multicolumn{2}{|c|}{2} & 42,5 \\
\hline 3 & 460 & 0,5 & \multicolumn{2}{|c|}{2} & 56 \\
\hline 4 & 460 & 1 & \multicolumn{2}{|c|}{1} & 60 \\
\hline 5 & 920 & 1 & \multicolumn{2}{|c|}{1} & 60,5 \\
\hline 6 & 920 & 0,5 & \multicolumn{2}{|c|}{2} & 58 \\
\hline Ech (YAG) & $\mathrm{E}\left(\mathrm{J} / \mathrm{cm}^{2}\right)$ & $V(\mathrm{~cm} / \mathrm{s})$ & $\tau(\mathrm{ms})$ & $\nu$ & A $\left(m^{2} / g\right)$ \\
\hline 7 & 45 & 0,5 & 20 & 2 & 65,5 \\
\hline 8 & 45 & $0(*)$ & 20 & 1 & 52 \\
\hline 9 & 45 & $0(*)$ & 5 & 1 & 45 \\
\hline
\end{tabular}

Ech $\left(\mathrm{CO}_{2}\right)$ : échantilions traitês au moyen du laser continu $\mathrm{CO}_{2}$ (samples treated with the continuons $\mathrm{CO}_{2}$ laser) ; Ech (YAG) : échantillons traités au moyen đu' laser YAG pulsé (samples treated with the YAG pulsed laser); $P, E$ : puissance ou énergie incidentes (incident power or energy); $V$ : vitesse de défilement de l'échantillon sous le faisceau (scanning speed) ; $t$ : temps d'interaction moyen d'un grain de graphite avec le faisceau (mean interaction time between the laser beam and a graphite grain); $\tau$ : durée d'un pulse (pulse time) ;

$\nu$ : nombre dirradiations subies par un grain de graphite $(\nu=2$ est un nombre moyen) (number of irradiations undergone by a graphite grain ( $\nu=2$ is an average number); $A$ : ajre spécifique des échantillons après exfoliation (resulting specific surface area) ;

(*) : traitement dans un tube à essai (experiment performed in a test-tube)

NB : diamètre du faisceau incident : $1 \mathrm{~cm}$ dans tous les cas (incident beam diameter : $1 \mathrm{~cm}$ ).

Surface spécifique obtenue par exfoliation à la flamme (environ $30 \mathrm{~m}^{2} / \mathrm{g}$ ) (specific surface area obtained by flame exfoliation : about $30 \mathrm{~m}^{2} / \mathrm{g}$ ).

\section{CARACTERISATION DE LA SURFACE APRES EXFOLIATION}

Cette caractérisation a été faite à partir de l'isotherme d'adsorption de krypton de $77,3 \mathrm{~K}$. (Se rapporter à (1) pour une description de la méthode).

\section{RESULTATS - DISCUSSION}

Les surfaces obtenues sont d'une homogénéité comparable à celle des graphites exfoliés par les méthodes habituelles (cf. (1)). Les aires spécifiques sont nettement supérieures à celles obtenues à la flamme (deux fois environ, cf. tableau 1).

L'ensemble des expériences donne une idée de l'importance dếs différentes variables considêrées. Les essais effectués avec le laser YAG (échantillons 7,8,9)montrent clairement l'importance du choc thermique et du temps d'interaction des grains du composé intercalaire avec le faisceau.

En conclusion, cette étude apporte la démonstration que le laser est un outil intéressant aussi bien pour la recherche des conditions optimales d'exfoliation du graphite que pour une meilleure compréhension du phénomène lui-même.

Remerciements : Nous remercions vivement hadame C. VIALLE pour son assistance technique. 


\section{BIBLIOGRAPHIE}

1. - A. THOMY, X. DUVAL, J. REGNIER, Surf. Sci. Reports 1, 1 (1981)

2. - X. DUVAL, A. THOMY, CR Acad. Sci, Paris, 259, 4007 (1964)

3. - A. THOMY, X. DUVAL, J. Chim. Phys. 66, 1966 (1969)

4. - R.E. STEPHENS, S.ROSS, S.P. WESSON, Carbon 11, 525 (1973)

5. - M.H. RICHMAN, A.P. LEVITT, E.S. DECESARE, Metallography 6, 497 (1973)

6. - M.B. DOWELL, Bienn. Conf. Carbon 12, Pittsburgh p.31 et 35 (1975)

7. - X. DUVAL, A. THOMY, Carbon 13, 242 (1975)

8. - C. MAZIERES, G. COLIN, J. JEGOUdeZ, R. SETTON, Carbon 14, 176 (1976)

9. - C. BOCKEL, A. THOMY, Carbon 19, 142 (1981)

10. - S.H. ANDERSON, D.D. CHUNG, Carbon 22, 253 (1984)

11. - R. ClARKE, P.M. HORN, S.E. NAGLER, T.F. ROSENBAUM, J. App1. Phys. 55, 1231 (1984)

12. - H. SPATZEK, Actes du Colloque International sur les Carbones, Baden-Baden, Springer Verlag p. 811 (1986)

13. - M. KLATT, G. FURDIN, A. HEROLD, N. DUPONT-PAVLOVSKY, Carbon 24, 731 (1986)

14. - M.S. DREsSelhaus, G. DRESSElhaus, Adv. Phys. 30, 139 (1981)

15. - A HEROLD in Intercalated Materials, Ed. F. LEVY, Reidel, Dordrecht p. 323 - 421 (1979)

16. - W. RÜDDORF, Zeit. Physik. Chem 45, 42 (1940)

17. - H. FUZELLIER, Thèse d'Etat, Nancy, CNRS AO 9580 (1974)

18. - M. KLATT, Thèse de l'Université de Nancy I (1985)

19. - D. ROYER, DEA Nancy (1986)

20. - G. FURDIN, Rapports Scientifiques internes

21. - "Le laser : principe et techniques d'application" - Coordonnateur : H.MAILLET Technique et Documentation - Lavoisier (1986)

22. - "Lasers et Industries de transformation" - Coordonnateur : A.B.VANNES, Technique et Documentation - Lavoisier (1986) 